Pacific Journal of Mathematics

THE ITERATED LIMIT CONDITION AND SEQUENTIAL 


\title{
THE ITERATED LIMIT CONDITION AND SEQUENTIAL CONVERGENCE
}

\author{
S. SIMONS
}

Let $F$ be a family of functions from an abstract set $X$ to a compact metric space $W$. This paper contains the following result: If $F$ satisfies the iterated limit condition (see $\$ 1$ below) then any function $f_{0}: X \rightarrow W$ that is pointwise limit of elements of $F$ can be expressed as the pointwise limit of a sequence extracted from $F$. Various generalizations are discussed, and applications are given to measure theory.

1. Notation and terminology. We suppose throughout this paper that $X$ is a nonvoid set and that $W$ is a topological space. We write $\left(W^{x}, p\right)$ for the set of all functions from $X$ to $W$, with the topology of pointwise convergence (product topology). We suppose also that $F \subset W^{x}$ and we write $F^{-}$for the closure of $F$ in $\left(W^{x}, p\right)$.

We say that $F$ satisfied the iterated limit condition if

$$
\lim _{m} \lim _{n} f_{n}\left(x_{m}\right)=\lim _{n} \lim _{m} f_{n}\left(x_{m}\right)
$$

whenever $\left\{f_{1}, f_{2}, \cdots\right\}$ is a sequence in $F$ and $\left\{x_{1}, x_{2}, \cdots\right\}$ is a sequence in $X$ for which all the limits exist.

2. Introduction. The following results about the iterated limit condition are known; if $X$ is a compact space, $W$ is a compact metric space and $F \subset G$, where $G$ is the set of continuous functions from $X$ to $W$, than (a), (b) and (c) are equivalent $[1,8.18, \mathrm{p} .76]$ and imply (d) $[1,8.20$, p. 78]

(a) $F$ satisfies the iterated limit condition

(b) $F^{-} \subset G$ (i.e., $F$ is relatively compact in $(G, p)$ )

(c) every sequence in $F$ has a cluster point in $(G, p)$

(d) every function in $F^{-}$is the $p$-limit of a sequence in $F$.

Now $(b)$ and (c) of the above conditions involve the fact that $X$ is a topological space, whereas (a) and (d) do not. In Theorem 1 of this paper we shall prove that (a) implies (d) without the hypothesis that $X$ is a topological space. The interest of this is that sequential convergence is important in the study of certain classes of functions that are not necessarily continuous (e.g., measurable functions). In later papers we shall be considering a number of applications of Theorem 1 to analysis. The reader who is primarily interested in these can pass over all parts of this paper that follow Theorem 1.

The result of Theorem 1 is essentially a combinatorial one and 
the proof we give of it is direct and elementary. This result can also be deduced from known results on continuous functions by using the Stone-Cech compactification, although it does not seem to be in print in any of the obvious places.

In Theorem 2 we shall prove a result similar to Theorem 1, only under less restrictive conditions on $W$. In the case of real functions, for instance, Theorem 2 leads to results about unbounded functions, whereas Theorem 1 only gives results about uniformly bounded families of functions. (Another possible approach would be to compactify the reals, but we do not discuss this.) In order to prove Theorem 2 it seems necessary to use a slightly modified form of the iterated limit condition.

In Theorem 3 and Corollary 4 we shall consider applications of our results to measure theory. Corollary 4 contains the following result: If $F \subset C[0,1]$ and $F$ is countably compact in the topology of pointwise convergence on $[0,1]$ then this topology on $F$ is identical with the topology of convergence in measure. We point out that the above conditions on $F$ do not imply that $F$ is a uniformly bounded set of functions. The above result is, in fact, a generalization of Egoroff's Theorem for continuous functions: if $f_{n} \in R^{[0,1]}$ and $f_{n} \rightarrow f_{o}$ in $p$ then $\left\{f_{0}, f_{1}, \cdots\right\}$ is compact in the topology of pointwise convergence on $[0,1]$.

\section{Results.}

Theorem 1. We suppose that $F$ satisfied the iterated limit condition and that $W$ is a compact metric space. Then:

(a) $\lim _{m} f_{n}(x)_{m}$ has $\lim _{m} \lim _{n} f\left(x_{m}\right)$ as a cluster point as $n \rightarrow \infty$

$\left\{\right.$ whenever $\left\{f_{1}, f_{2}, \cdots\right\}$ is a sequence in $F$ and $\left\{x_{1}, x_{2}, \cdots\right\}$

is a sequence in $X$ for which all the limits exist;

(b) if $f_{0} \in F^{-}$then $f_{0}$ is the p-limit of a sequence in $F$.

THeorem 2. If $F$ satisfies (1) above and $W$ is a metric space and the union of a countable family of precompact subsets then Theorem 1 (b) is still true.

THeOREm 3. Let $S$ be a $\sigma$-field of subsets of $X, F$ a family of real, $S$-measurable functions on $X$ that satisfies (1), and $f_{0} \in R^{X}$ be the p-limit of a net $f_{\alpha}$ in $F$. Then

(a) $f_{0}$ is $S$-measurable,

(b) if $\mu$ is a finite measure on $S$ then $f_{\alpha} \rightarrow f_{0}$ in $\mu$-measure, and

(c) if $\mu$ is a finite measure on $S$ and there exists a $\mu$-integrable function $g$ on $X$ such that $\left|f_{\alpha}\right| \leqq g[\mu]$ for all $\alpha$ then $f_{0}$ is $\mu$-integrable 
and $f_{\alpha} \rightarrow f_{0}$ in $\mu$-mean.

REMARKs. The results of Theorem 3 are true if $F$ is a uniformly bounded set of real functions that satisfies the iterated limit condition (from Theorem 1(a)). However, we shall be proving deeper results in a later paper.

If we omit the condition that $F$ satisfies (1) then all the results of Theorem 3 are false. Suppose, for example, we take $X=(0,1)$, $S=$ Lebesgue measurable sets and $\mu=$ Lebesgue measure. Let $\alpha$ run through the finite subsets of $X$, directed by $\supset$. If $f_{\kappa}$ is the characteristic function of $\alpha$ then $f_{\alpha} \rightarrow 1$ in $p$ but $\int f_{\alpha} d \mu=0 \nrightarrow 1=\int 1 d \mu$. If $h$ is any nonmeasurable function then $f_{\alpha} h \rightarrow h$ in $p$, but each function then $f_{\alpha} h \rightarrow h$ in $p$, but each function $f_{\alpha} h$ is measurable.

This brings us to another point: if $f_{\alpha}$ are as above and $h$ is the function $t \rightarrow 1 / t$ then

$$
\text { for each } \left.\alpha,\left|f_{\alpha} h\right|=0 \mid \mu\right]
$$

but there is no measurable set $E \subset(0,1)$ and integrable function $g$ on $(0,1)$ such that

$$
\mu((0,1) \backslash E)=0
$$

and

$$
\left|f_{\alpha} h(x)\right| \leqq g(x) \text { for all } \alpha \text { and for all } x \in E .
$$

On the other hand, if $\left\{f_{1}, f_{2}, \cdots\right\}$ is a sequence of S-measurable functions on $X, \mu$ is a finite measure on $S$ and there exists an integrable $g$ such that

$$
\text { for each } \left.n,\left|f_{n}\right| \leqq g \mid \mu\right]
$$

then there exists $E \in S$ such that

$$
\mu(X \backslash E)=0
$$

and

$$
\left|f_{n}(x)\right| \leqq g(x) \text { for all } n \text { and for all } x \in E \text {. }
$$

(This is immediate since the union of a countable family of sets of measure zero has measure zero). This suggests the following

Problem. If $(X, S), F$ and $\mu$ are as in Theorem $3(\mathrm{~b})$ and there exists a $\mu$-integrable function $g$ on $X$ such that $|f| \leqq g[\mu]$ for all $f \in F$ then does there necessarily exist $E \in S$ with $\mu(X \backslash E)=0$ and a $\mu$-integrable function $g^{\prime}$ on $X$ such that 


$$
|f(x)| \leqq g^{\prime}(x) \text { for all } f \in F \text { and for all } x \in E \text { ? }
$$

CoRollary 4. If $X$ is a countably compact topological space, $F$ is a countably compact subset of $\left(R^{x}, p\right)$ and $F$ consists of continuous functions, $\mu$ is a finite measure on $X$ such that $\int f d \mu$ exists for all $f \in F$ and such that

$$
f, g \in F \text { and } f \neq g \text { imply that } f \neq g[\mu]
$$

then $p$ and the topology of convergence in $\mu$-measure coincide on $F$.

These conclusions are true, in particular, if $X$ is compact Hausdorff, $\mu$ is a regular Borel measure on $X$ with support $X$ and $F$ is any $p$-countably compact set of continuous functions.

4. Proofs. We write $d$ for the metric on $W$.

Proof of Theorem 1.

(a) We suppose that $\left\{f_{1}, f_{2}, \cdots\right\}$ is a sequence in $F$ and $\left\{x_{1}, x_{2}, \cdots\right\}$ is a sequence in $X$ for which $\lim _{m} \lim _{n} f_{n}\left(x_{m}\right)$ exists and, for each $n$, $\lim _{m} f_{n}\left(x_{m}\right)$ exists. Since $\mathrm{W}$ is compact metric, there exist integers $n(1)<n(2)<\cdots$ such that $\lim _{i} \lim _{m} f_{n(i)}\left(x_{m}\right)$ exists. On the other hand, for each $m, \lim _{i} f_{n(i)}\left(x_{m}\right)=\lim _{n} f_{n}\left(x_{m}\right)$ since $n(i) \rightarrow \infty$ as $i \rightarrow \infty$, hence

$$
\lim _{m} \lim _{i} f_{n(i)}\left(x_{m}\right)=\lim _{m} \lim _{n} f_{n}\left(x_{m}\right) .
$$

Appplying the iterated limit condition to $\left\{f_{n(1)}, f_{n(2)}, \cdots\right\}$ and $\left\{x_{1}, x_{2}, \cdots\right\}$ we find that

$$
\lim _{i} \lim _{m} f_{n(i)}\left(x_{m}\right)=\lim _{m} \lim _{n} f_{n}\left(x_{m}\right)
$$

and so (1) is satisfied, completing the proof of (a).

(b) We find $f_{1}, Z_{1}, f_{2}, Z_{2}, \cdots$ inductively, where $f_{1}, f_{2}, \cdots \in F$ and $Z_{1}, Z_{2}, \cdots$ are finite subsets of $X$, such that

$$
\left\{\begin{array}{l}
\text { for each } s=1,2, \cdots \text { and for each } x \in X \text { there exists } \\
z(x, s) \in Z_{s} \text { such that } \sup _{0 \leqq r \leqq s} d\left(f_{r}(z(x, s)), f_{r}(x)\right)<\frac{1}{s}
\end{array}\right.
$$

$$
\text { for each } s=2,3, \cdots Z_{s-1} \subset Z_{s}
$$

and

(4) for each $s=2,3, \cdots, z \in Z_{s-1}$ implies that $d\left(f_{s}(z), f_{0}(z)\right)<\frac{1}{s}$.

We perform the induction as follows. We first choose $f_{1} \in F$ 
arbitrarily ( $F$ is nonvoid since $f_{0} \in F^{-}$). Having chosen

$$
f_{1}, Z_{1}, \cdots, Z_{n-1}, f_{n}
$$

we observe that if the function $g: X \rightarrow W^{n+1}$ is defined by

$$
g(x)=\left(f_{0}(x), \cdots, f_{n}(x)\right)
$$

then $g(X)$ is precompact (since $W^{n+1}$ is compact). It follows by using the sup metric on $W^{n+1}$, that there exists $Z_{n}$ satisfying $(2) \quad(s=n)$ and, by taking the union with $Z_{n-1}$, satisfying $(3)(s=n)$.

Having chosen $f_{1}, Z_{1}, \cdots, f_{n-1}, Z_{n-1}$ it is immediate since $f_{0} \in F^{-}$ that there exists $f_{n} \in F$ that satisfies $(4)(s=n)$.

We show that $f_{n} \rightarrow f_{0}$ in $p$. If this were false there would exist $x \in X, \varepsilon>0$ and $1 \leqq s(1)<s(2)<\cdots$ such that

$$
\text { for } n=1,2, \cdots \quad d\left(f_{s(n)}(x), f_{0}(x)\right) \geqq \varepsilon .
$$

For any $n$, if $m$ is sufficiently large (specifically, if $m_{d} \geqq n$ ) then from (2),

$$
d\left(f_{s(n)}(z(x, s(m))), f_{s(n)}(x)\right)<\frac{1}{s(m)}
$$

and so, for each $n$,

$$
\lim _{m} f_{s(n)}(z(x, s(m)))=f_{s(n)}(x) .
$$

Similarly

$$
\lim _{m} f_{0}(z(x, s(m)))=f_{0}(x) .
$$

For any $m$, if $n>m$ then, from (3) and (4),

$$
d\left(f_{s(n)}(z(x, s(m))), f_{0}(z(x, s(m)))\right)<\frac{1}{s(n)} ;
$$

thus

$$
\lim _{n} f_{s(n)}(z(x, s(m)))=f_{0}(z(x, s(m)) .
$$

From (8) and (9),

$$
\lim _{m} \lim _{n} f_{s(n)}(z(x, s(m)))=f_{0}(x)
$$

thus (5) and (7) contradict (1) (that is, part (a) of this theorem) with $f_{n}$ replaced by $f_{s(n)}$ and $x_{m}$ replaced by $z(x, s(m))$. This completes the proof of (b).

Proof of Theorem 2. We first observe that if $f_{0}$ and each of the functions in $F$ have a precompact range then the proof given in 
Theorem $1(b)$ is still valid (the fact that $W$ is compact is used in Theorem 1(b) to show that (1) follows from the iterated limit condition $-g(X)$ is still precompact in $W^{n+1}$, being contained in

$$
\left.f_{0}(X) \times f_{1}(X) \times \cdots \times f_{n}(X) .\right) .
$$

We turn now to the general case and suppose that $W=U_{n \geqq 1} K_{n}$, where $K_{1}, K_{2}, \cdots$ are precompact in $W$ and $K_{1} \subset K_{2} \subset \cdots$. We find $f_{1}, Z_{1}, f_{2}, Z_{2}, \cdots$ inductively, where $f_{1}, f_{2}, \cdots \in F$ and $Z_{1}, Z_{2}, \cdots$ are finite subsets of $X$, such that

$$
\left\{\begin{array}{l}
\text { for each } s=1,2, \cdots \text { and for each } x \in X \text { there exists } \\
z(x, s) \in Z_{s} \text { such that } \sup \left\{d\left(f_{r}(z(x, s)), f_{r}(x)\right): 0 \leqq r \leqq s\right. \\
\left.\qquad f_{r}(x) \in K_{s}\right\} \leqq \frac{1}{s}
\end{array}\right.
$$

and (3) and (4) are satisfied as before.

We perform the induction as follows. We first choose $f_{1} \in F$ arbitrarily. Having chosen $f_{1}, Z_{1}, \cdots, f_{n-1}, Z_{n-1}$ it follows (as before) that, since $f_{0} \in F^{-}$, there exists $f_{n} \in F$ that satisfies (4) $(s=n)$. We now come to the point where our proof differs markedly from that of Theorem 1 (b). We suppose that $f_{1}, Z_{1}, \cdots, Z_{n-1}, f_{n}$ have been chosen and we discuss the choice of $Z_{n}$. For any $I \subset\{0,1, \cdots, n\}$ we write

$$
V=\cap_{r \in I} f_{r}^{-1}\left(K_{n}\right)
$$

and define the map $g: V \rightarrow W^{I}$ by

$$
(g(x))_{r}=f_{r}(x), \quad(r \in I) .
$$

Since $g(V) \subset\left(K_{n}\right)^{I}, g(V)$ is precompact in $W^{I}$ and there exists a finite subset $Y$ of $V$ such that

for all $x \in V$ there exists $y \in Y$ such that $\left.\sup _{r \in I} d\left(f_{r}\right)(x), f_{r}(y)\right)<\frac{1}{s}$.

We choose $Z_{n}$ so that $Z_{n} \supset Z_{n-1}$ and also $Z_{n}$ contains all the sets $Y$, as $I$ varies over all the subsets of $\{0,1, \cdots, n\}$. It follows that $Z_{n}$ satisfies (10) and (3) $(s=n)$.

The rest of the proof proceeds as before, except that "specifically if $m \geqq n$ " in the line preceding (6) is replaced by "specifically, if $m \geqq n$ and $m$ is so large that $K_{s(m)} \ni f_{s(n)}(x)$."

\section{Proof of Theorem 3.}

(a) This follows from Theorem $2-f_{0}$ is the limit of a sequence extracted from $F$.

(b) If this were false then, for some $\varepsilon>0$, we could find a cofinal subset $\{\beta\}$ of $\{\alpha\}$ such that, for each $\beta$, 


$$
\int \frac{\left|f_{\beta}-f_{0}\right|}{1+\left|f_{\beta}-f_{0}\right|} d \mu \geqq \varepsilon
$$

Then, from Theorem 2, we could find $\beta(1), \beta(2), \cdots$ such that $f_{\beta(n)} \rightarrow f_{0}$ in $p$. This is impossible since, from Egoroff's Theorem, pointwise convergence of a sequence implies convergence in measure.

(c) The proof of this is similar to that of (b): for a dominated sequence pointwise convergence implies convergence in mean.

Proof of Corollary 4. If $f_{1}, f_{2}, \cdots$ and $x_{1}, x_{2}, \cdots$ are as in (1), $f \in F$ is a cluster point of $\left\{f_{1}, f_{2}, \cdots\right\}$ in $\left(R^{x}, p\right)$ and $x$ is a cluster point of $\left\{x_{1}, x_{2}, \cdots\right\}$ in $X$ then

$$
\lim _{m} f_{n}\left(x_{m}\right)=f_{n}(x) \quad \text { since } f_{n} \text { is continuous }
$$

and

$$
\lim _{m} \lim _{n} f_{n}\left(x_{m}\right)=\lim _{m} f\left(x_{m}\right)=f(x) \text { since } f \text { is continuous . }
$$

Thus $F$ satisfies (1). The result now follows from Theorem 3 (b) and the theorem that if $(F, p)$ is a countably compact space, $(F, m)$ is a metrizable space and $m$ is coarser than $p$, then $m$ and $p$ are identical. (If $A$ is closed in $(F, p)$ then $A$ is countably compact in $(F, p)$, hence countably compact in $m$; any countably compact subset of a metric space is closed hence $A$ is closed in $(F, m)$ ).

5. Further comments and an example. If $W$ is any Hausdorff topological space, condition (1) implies that $F$ satisfies the iterated limit condition. So also does the following condition:

$\operatorname{Lim}_{n} f_{n}\left(x_{m}\right)$ converges to $\lim _{n} \lim _{m} f_{n}\left(x_{m}\right)$ as $m \rightarrow \infty$ whenever $\left\{f_{1}, f_{2}, \cdots\right\}$ is a sequence in $F$ and $\left\{x_{1}, x_{2}, \cdots\right\}$ is a sequence in $X$ for which all the limits exist.

If $F$ is a family of real functions on $X$ and $F$ satisfies the iterated limit condition we cannot assert that every $f_{0} \in F^{-}$is the $p$-limit of a sequence in $F$ if the functions in $F$ are not uniformly bounded, even if each $f \in F$ is bounded. In such a case it follows from Theorem 2 that $F$ does not satisfy (1). (Cf. Theorem 1(a)). It can also happen that $F$ satisfies the iterated limit condition and that every $f_{0} \in F^{-}$is the limit of a sequence in $F$ yet $F$ does not satisfy (1). Both these points are illustrated by the following example.

Notation. Let $X$ be any infinite set. For each finite subset $Y$ of $X$ let $\nu(Y)$ denote the number of elements in $Y$. We write $f_{Y}$ for the function on $X$ defined by 


$$
f_{Y}(x)= \begin{cases}0 & (x \in X) \\ \nu(Y) & (x \in X \backslash Y)\end{cases}
$$

Lemma. We suppose that $Y(1), Y(2), \cdots$ are nonvoid subsets of $X$ and $x_{1}, x_{2}, \cdots \in X$ are such that, for each $x \in X$, eventually $x_{m} \neq x$.

(a) If $\lim _{n} \lim _{m} f_{Y(n)}\left(x_{m}\right)=r$ then $r \geqq 1$ and $r=\lim _{n} \nu(Y(n))$

(b) If $\lim _{n} f_{Y(n)}\left(x_{m}\right)=0$ for arbitrarily large values of $m$ then $\nu(Y(n) j \rightarrow \infty$ as $n \rightarrow \infty$.

(c) If $\lim _{n} f_{Y(n)}\left(x_{m}\right)>0$ then its value is $\lim _{n} \nu(Y(n))$

Proofs. (a) For any $n, x_{m}$ is eventually outside the finite set $Y(n)$ hence $\lim _{m} f_{Y(n)}\left(x_{m}\right)=\nu(Y(n))$. This proves (a).

(b) For any positive integer $p$ there exist $m(1), m(2) \cdots m(p)$ such that $x_{m(1)}, \cdots, x_{m(p)}$ are distinct and, for each $i=1 \cdots p$,

$$
\lim _{n} f_{Y(n)}\left(x_{m(i)}\right)=0 .
$$

Hence, for each $i=1 \cdots p$,

$$
f_{Y(n)}\left(x_{m(i)}\right)=0 \quad \text { for all sufficiently large } n
$$

and so

$$
x_{m(i)} \in Y(n) \quad \text { for all sufficiently large } n \text {. }
$$

Thus

$$
\left\{x_{m(1)}, \cdots, x_{m(p)}\right\} \subset Y(n) \quad \text { for all sufficiently large } n
$$

and so

$$
\nu(Y(n)) \geqq p \quad \text { for all sufficiently large } n \text {. }
$$

This proves $(b)$.

(c) If $\lim _{n} f_{Y(n)}\left(x_{m}\right)>0$ then, for all sufficiently large $n, f_{Y_{(n)}}\left(x_{m}\right)>0$ and so $f_{Y(n)}\left(x_{m}\right)=\nu(Y(n))$. This proves $(c)$.

THEOREM. If $F=\left\{f_{Y}\right\}$, with $Y$ ranging through the nonvoid finite subsets of $X$, then

(a) $F$ satisfies the iterated limit condition (and in fact the stronger condition (11)).

(b) $0 \in F^{-}$.

(c) If $X$ is uncountable there is no sequence in $F$ that converges to 0 in $p$.

(d) If $X$ is countably infinite every $f_{0} \in F^{-}$is the p-limit of a sequence in $F$, but $F$ does not satisfy (1).

Proofs. (a) We prove that $F$ satisfies (11): if $\lim _{n} \lim _{m} f_{Y(n)}\left(x_{m}\right)=r$ 
and $l(m)=\lim _{n} f_{Y(n)}\left(x_{m}\right)$ exists for all $m$ then, from (a) of the Lemma, $r=\lim _{n} \nu(Y(n))$. Thus, from (b) of the Lemma, $l(m)>0$ for all sufficiently large values of $m$. It follows from (c) that $l(m)=r$ for all sufficiently large values of $m$. This proves (a).

(b) is immediate, and (c) follows from the observation that the union of a countable number of finite sets is countable.

The first assertion of (d) follows from the fact that $p$ is now a metrizable topology. If $x_{1}, x_{2}, \cdots$ are distinct elements of $X$ and $Y(n)=\left\{x_{1}, \cdots, x_{n}\right\}$ then $\lim _{m} \lim _{n} f_{Y(n)}\left(x_{m}\right)=\lim _{m} 0=0$ and

$$
\lim _{m} f_{Y(n)}\left(x_{m}\right)=n
$$

for all $n$, thus $F$ does not satisfy (1).

\section{REFERENCES}

1, J. L. Kelly, I. Namioka, et. al. Linear Topological Spaces, van Nostrand, 1963.

Received December 21, 1965.

UNIVERSity of California, SANTA Barbara 



\section{PACIFIC JOURNAL OF MATHEMATICS}

\section{EDITORS}

\section{H. SAMELSON}

Stanford University

Stanford, California

J. P. JANS

University of Washington

Seattle, Washington 98105
J. DUGUNDJI

University of Southern California Los Angeles, California 90007

RICHARD ARENS

University of California

Los Angeles, California 90024

\section{ASSOCIATE EDITORS}
E. F. BECKENBACH
B. H. NEumanN
F. WOLF
K. YosidA

\section{SUPPORTING INSTITUTIONS}

\author{
UNIVERSITY OF BRITISH COLUMBIA \\ CALIFORNIA INSTITUTE OF TECHNOLOGY \\ UNIVERSITY OF CALIFORNIA \\ MONTANA STATE UNIVERSITY \\ UNIVERSITY OF NEVADA \\ NEW MEXICO STATE UNIVERSITY \\ OREGON STATE UNIVERSITY \\ UNIVERSITY OF OREGON \\ OSAKA UNIVERSITY \\ UNIVERSITY OF SOUTHERN CALIFORNIA
}

\author{
STANFORD UNIVERSITY \\ UNIVERSITY OF TOKYO \\ UNIVERSITY OF UTAH \\ WASHINGTON STATE UNIVERSITY \\ UNIVERSITY OF WASHINGTON \\ AMERICAN MATHEMATICAL SOCIETY \\ CHEVRON RESEARCH CORPORATION \\ TRW SYSTEMS \\ NAVAL ORDNANCE TEST STATION
}

Mathematical papers intended for publication in the Pacific Journal of Mathematics should be typewritten (double spaced). The first paragraph or two must be capable of being used separately as a synopsis of the entire paper. It should not contain references to the bibliography. Manuscripts may be sent to any one of the four editors. All other communications to the editors should be addressed to the managing editor, Richard Arens at the University of California, Los Angeles, California 90024.

50 reprints per author of each article are furnished free of charge; additional copies may be obtained at cost in multiples of 50 .

The Pacific Journal of Mathematics is published monthly. Effective with Volume 16 the price per volume ( 3 numbers) is $\$ 8.00$; single issues, $\$ 3.00$. Special price for current issues to individual faculty members of supporting institutions and to individual members of the American Mathematical Society: $\$ 4.00$ per volume; single issues $\$ 1.50$. Back numbers are available.

Subscriptions, orders for back numbers, and changes of address should be sent to Pacific Journal of Mathematics, 103 Highland Boulevard, Berkeley 8, California.

Printed at Kokusai Bunken Insatsusha (International Academic Printing Co., Ltd.), 7-17, Fujimi 2-chome, Chiyoda-ku, Tokyo, Japan.

PUBLISHED BY PACIFIC JOURNAL OF MATHEMATICS, A NON-PROFIT CORPORATION

The Supporting Institutions listed above contribute to the cost of publication of this Journal, but they are not owners or publishers and have no responsibility for its content or policies. 


\section{Pacific Journal of Mathematics

Vol. 22, No. $3 \quad$ March, 1967

Wai-Mee Ching and James Sai-Wing Wong, Multipliers and $H^{*}$

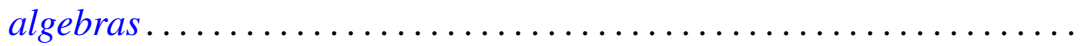

P. H. Doyle, III and John Gilbert Hocking, A generalization of the Wilder

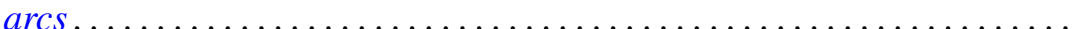

Irving Leonard Glicksberg, A Phragmén-Lindelöf theorem for function

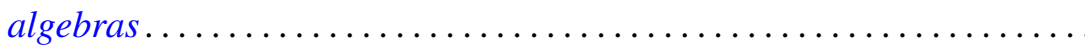

E. M. Horadam, A sum of a certain divisor function for arithmetical semi-groups ..................................... 407

V. Istrăţescu, On some hyponormal operators ................... 413

Harold H. Johnson, The non-invariance of hyperbolicity in partial

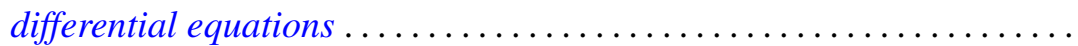

Daniel Paul Maki, On constructing distribution functions: A bounded denumerable spectrum with $n$ limit points................... 431

Ronald John Nunke, On the structure of Tor. II .................... 453

T. V. Panchapagesan, Unitary operators in Banach spaces ............. 465

Gerald H. Ryder, Boundary value problems for a class of nonlinear differential equations ................................. 477

Stephen Simons, The iterated limit condition and sequential convergence .................................... 505

Larry Eugene Snyder, Stolz angle convergence in metric spaces ......... 515 Sherman K. Stein, Factoring by subsets ................... 523

Ponnaluri Suryanarayana, The higher order differentiability of solutions of abstract evolution equations . . .

Leroy J. Warren and Henry Gilbert Bray, On the square-freeness of Fermat and Mersenne numbers ............................... 563

Tudor Zamfirescu, On l-simplicial convexity in vector spaces........... 565 Eduardo H. Zarantonello, The closure of the numerical range contains the spectrum 